Research Paper

\title{
Short-Term High-Dose Vitamin C and E Supplementation Attenuates Muscle Damage and Inflammatory Responses to Repeated Taekwondo Competitions: A Randomized Placebo-Controlled Trial
}

\author{
Chun-Chung Chou ${ }^{1}$, Yu-Chi Sung $2,{ }^{*}$, Glen Davison ${ }^{3,},{ }^{*}$, Chung-Yu Chen ${ }^{4,}$, , Yi-Hung Liao ${ }^{5,}{ }^{凶}$ \\ 1. Physical Education Office, National Taipei University of Technology, Taipei City, Taiwan \\ 2. Department of Chinese Martial Arts, Chinese Culture University, Taipei City, Taiwan \\ 3. Endurance Research Group, School of Sport and Exercise Sciences, University of Kent, Medway Campus, Chatham Maritime, UK \\ 4. Department of Exercise and Health Science, University of Taipei, Taipei City, Taiwan \\ 5. Department of Exercise and Health Science, National Taipei University of Nursing and Health Sciences, Taipei City, Taiwan \\ "Sung Y.-C., Davison G., and Chen C.-Y. contributed equally to this work.
}

$\square$ Corresponding author: Yi-Hung Liao, P.T., Ph.D., Department of Exercise and Health Science, National Taipei University of Nursing and Health Sciences, No.365, Ming-Te Road, Peitou District, Taipei City 11219, Taiwan. E-mail: yihungliao.henry@gmail.com; Phone: +886-2-28227101 ext. 7707; Fax: +886-2-2875-3383

(c) Ivyspring International Publisher. This is an open access article distributed under the terms of the Creative Commons Attribution (CC BY-NC) license (https://creativecommons.org/licenses/by-nc/4.0/). See http://ivyspring.com/terms for full terms and conditions.

Received: 2018.03.28; Accepted: 2018.07.01; Published: 2018.07.30

\begin{abstract}
Background: Exercise-induced muscle damage during intensive sport events is a very common issue in sport medicine. Therefore, the purpose is to investigate the effects of short-term high-dose vitamin $\mathrm{C}$ and $\mathrm{E}$ supplementation on muscle damage, hemolysis, and inflammatory responses to simulated competitive Olympic Taekwondo (TKD) matches in elite athletes.

Methods: Using a randomized placebo-controlled and double-blind study design, eighteen elite male TKD athletes were weight-matched and randomly assigned into either a vitamin $C$ and $E$ group $(V i t \mathrm{C}+\mathrm{E} ; \mathrm{N}=9$ ) or placebo group $(\mathrm{PLA} ; \mathrm{N}=9)$ ). Vit $\mathrm{C}+\mathrm{E}$ or PLA supplements were taken daily (Vit C+E: $2000 \mathrm{mg} / \mathrm{d}$ vitamin C; $1400 \mathrm{U} / \mathrm{d}$ vitamin E) for 4 days (3 days before and on competition day) before taking part in 4 consecutive TKD matches on a single day. Plasma samples were obtained before each match and 24-hours after the first match for determination of markers of muscle damage, hemolysis, and systemic inflammatory state.

Results: Myoglobin was lower in the Vit C+E group, compared to PLA, during the match day (area under curve, AUC $-47.0 \%$ vs. PLA, $p=0.021$ ). Plasma creatine kinase was lower in the Vit $C+E$ group (AUC $-57.5 \%$ vs. PLA, $p=0.017$ ) and hemolysis was lower in the Vit $C+E$ group (AUC $-40.5 \%$ vs. PLA, $P=0.034)$.

Conclusions: We demonstrated that short-term (4-days) vitamin $C$ and $E$ supplementation effectively attenuated exercise-induced tissue damage and inflammatory response during and after successive TKD matches.
\end{abstract}

Key words: muscle damage, inflammation, antioxidant, myoglobin, hemolysis

\section{Background}

Vitamin E (a-tocopherol) and vitamin C (ascorbic acid) are common antioxidants found in natural foods, both of which have strong antioxidant capacity to prevent oxidative damage [1, 2]. Vitamin E is a lipid-soluble antioxidant and can quench free radicals that may attack membrane phospholipid or lipoprotein [2]. Vitamin $\mathrm{C}$ is a water-soluble antioxidant and can directly quench free radicals in aqueous milieu (including within cells), but may also recycle vitamin $\mathrm{E}$ and other antioxidants by returning 
them to a reduced state, and thereby suppress oxidative damage [1]. The addition of vitamin E into dietary food has been demonstrated to protect against oxidative stress and muscle damage resulting from high-intensity exercise training [3]. Furthermore, high-dose vitamin $C$ supplementation has been shown to reduce eccentric exercise-induced muscle soreness and damage [4]. The combination of vitamins $C$ and $E$ has been suggested to produce synergistic effects and yield greater antioxidant capacities because vitamin $\mathrm{E}$ exists only in lipid-containing structures whereas vitamin $\mathrm{C}$ can exist in all aqueous milieu and can also recycle vitamin E (and other antioxidants) [5-7]. Several lines of evidence have revealed that provision of vitamin $C$ (dose range: $200-3000 \mathrm{mg} /$ day) or vitamin E (dose range: 400-1200 IU / day) for 2-4 weeks prior to an acute bout of high-intensity exercise can attenuate exercise-induced muscle tissue damage [4, 5, 8], cellular oxidative damage [8], and impairments in muscular contractility [9].

Some recent studies have shown that daily supplementation with high doses of vitamin C, vitamin $\mathrm{E}$, or other antioxidants during endurance training can blunt beneficial training-induced physiological adaptations, such as muscle oxidative capacity and mitochondrial biogenesis [10, 11]. However, others have reported no negative effects on physiological outcomes or training adaptation when training is sufficiently intensive/stressful [12, 13]. Despite this there is a strong possibility (and growing body of evidence) to suggest that long-term provision of antioxidants may attenuate exercise-induced training adaptions, likely due to the effects on free radical mediated intracellular processes. However, during competition when repeated bouts are required with relatively short recovery intervals (and possibly on consecutive days) the chronic training adaptations are not important, but rather the ability to recover and perform at maximal capacity is the main concern. In this situation short term beneficial effects on muscle damage, and inflammatory responses $[4,5,8,9]$ may be advantageous to repeated performance ability of athletes. However, because of the reported negative effects from chronic high intakes, shorter periods of supplementation may be more desirable.

Taekwondo is a traditional Korean military-based martial art, which was introduced as an official Olympic sports discipline in 2000 [14]. This combative sport requires athletes to perform many high-intensity movements, including intensive side-kicks, speedy defending movements, rapid jumping, fast spinning-kicks, and powerful punching [14-17]. According to the Olympics and other international level Taekwondo competition rules, the athletes have to continuously compete for at least 4 matches in one day to progress to the final championship game, and may be required to endure consecutive competition schedules. Intensive high-intensity exercise or competition can markedly increase acute muscle micro-damage and raise physiological stresses due to the high exercise intensity and large amount of eccentric movements $[18,19]$. To our knowledge, there are very limited studies using a simulated competitive match model in elite combative athletes. Consequently, further research in this area will provide important practical information of benefit for coaches and athletes.

The aim of this study was to determine the effects of short-term (4 days) high-dose vitamin $C$ and E supplementation (Vitamin C: $2000 \mathrm{mg} / \mathrm{d}$; Vitamin E: $1400 \mathrm{U} / \mathrm{d}$ ) on exercise-induced muscle damage and inflammatory markers during combative competition (mimicking Olympic games competition timeframe) in elite TKD athletes.

\section{Methods and materials}

\section{Participants and ethical statement}

Eighteen elite male TKD athletes volunteered to participate in this study. They were all black-belt holders and categorized as the Division I athletes and currently active at national or international levels during the time of the experiment. All participants were weight-matched and randomly assigned into either a Vitamin $C$ and $E$ supplement group (Vit $C+E$; $\mathrm{N}=9$, age: $21.0 \pm 0.3$ years, weight: $67.9 \pm 3.0 \mathrm{~kg}$, height: $175.8 \pm 2.1 \mathrm{~cm}$, BMI: $21.9 \pm 0.7 \mathrm{~kg} / \mathrm{m}^{2}$ ) or a placebo group (PLA; $\mathrm{N}=9$, age: $21.3 \pm 0.6$ years, weight: $71.5 \pm 3.1 \mathrm{~kg}$, height: $178.1 \pm 2.7 \mathrm{~cm}$, BMI: 22.4 $\left.\pm 0.5 \mathrm{~kg} / \mathrm{m}^{2}\right)$, and there were no significant differences among all anthropometric parameters between groups. The weight category of collegiate participants were classified in accordance with the Olympic weight class rules of TKD discipline as follow: $\leq 58 \mathrm{~kg}$ $(\mathrm{n}=2),>58$ to $\leq 68 \mathrm{~kg}(\mathrm{n}=6),>68$ to $\leq 80 \mathrm{~kg}(\mathrm{n}=8)$, $>80 \mathrm{~kg}(\mathrm{n}=2)$. Prior to the participant recruitment, the study had been approved by the Institute Review Board of the University of Taipei (IRB-2015-009), and this study was conducted according to the principles expressed in the last version of the Declaration of Helsinki. Moreover, the purpose and experimental procedures were carefully explained to the participants by the research team, and all participants completed an informed consent form prior to participating.

\section{Experimental design}

A randomized, placebo-controlled and double-blind study design was used. One week before the experiment day, all participants reported to the 
laboratory for recording of their basic characteristics, including general anthropometric measurements (weight, height, body fat \%), and then they were randomly assigned to either Vit $\mathrm{C}+\mathrm{E}(\mathrm{N}=9)$ or PLA $(\mathrm{N}=9)$ groups according to the weight-matched results. For physical activity and dietary control, we used the self-controlled methods commencing one-week before the experiment by instructing participants to maintain light-intensity physical activity to minimize the impacts from prior exercise and by providing general dietary guidelines (e.g., so that dietary intake comprised $\sim 60 \%$ of energy from carbohydrate, $10-15 \%$ from protein, and $15-25 \%$ from fat). The participants all received a 4 day supply of their respective supplements (Vit $\mathrm{C}+\mathrm{E}$ or placebo) but were blinded to group allocation. They were instructed to consume the supplements for the 3 days before and morning of the competition day. A blinded research team member was responsible for providing supplements to the participants according to their group assignment, and logs were also recorded to ensure their compliance with supplement consumption. During the entire experiment period, the participants did not use any forms of nutritional supplements for at least 3 months prior to the experiment, and all were informed not to engage in moderate to heavy exercise for $72 \mathrm{~h}$ before the simulated competition day.

\section{Procedures of simulated Olympic style taekwondo competition}

All participants competed against an opponent within their corresponding Olympic weight categories and matched experience levels to ensure the intensity of simulated competitions. The simulated TKD fighting matches were performed within an $8 \mathrm{~m} \times 8 \mathrm{~m}$ rubber mat octagonal competition area according to the last version of the regulations and rules of Olympic TKD game by the World Taekwondo Federation (WTF). Each match consisted of 3 rounds of TKD fighting ( $2 \mathrm{~min}$ per round separated by a 1 min break), and all participants were required to compete for 4 consecutive matches against different opponents during the simulated competition day. The experimental procedure of the simulated Olympic style Taekwondo competition is illustrated in Figure 1A. On the day of simulated TKD competition, fasting blood samples were collected from the participants at 0700 (Pre-M1 blood) after reporting to the stadium, and then they were provided a standard breakfast $(528 \mathrm{kcal}$; see Table 1 for the detailed macronutrient intake during TKD match day) with the morning Vit $\mathrm{C}+\mathrm{E}$ or PLA supplements at 0720 . Thereafter, the participants underwent a total of four serial TKD matches at 0830
(Match \#1), 1030 (Match \#2), 1400 (Match \#3), and 1630 (Match \#4), and pre-match blood samples were collected $10 \mathrm{~min}$ prior to each match (i.e. Pre-M2 blood, Pre-M3 blood, Pre-M4 blood). The intervals between matches ranged from 2 to 3.5 hours according to the competition arrangements. A wireless heart rate monitor (Polar ${ }^{\circledR}$ RS800CX ${ }^{\mathrm{TM}}$; Polar Electro Inc., Lake Success, NY, USA) was used to periodically record the heart rate (HR) during rest and TKD simulated matches, and fingertip blood samples were also collected immediately post-matches for determination of blood lactate levels by lactate meter (Edge Blood Lactate Monitoring System, ApexBio Inc., Taipei City, Taiwan). Moreover, standardized snacks ( 400-500 kcal/per snack; see Table 1 for detail) were provided immediately after each match, and the standard dinner $(1110 \mathrm{kcal}$; see Table 1 for detail) and evening supplements were provided at 1730 after the final match. The standardized post-match snacks and dinner were requested to be finished within $30 \mathrm{~min}$. The total energy consumption, including regular meals and snacks, during the day of simulated TKD matches was approximately $3000 \mathrm{kcal}$ (see Table 1 for macronutrient distribution). Finally, the Post-24 h fasting blood samples were collected the following morning at 0700 .

Table 1. Macronutrient and energy intake during competition day

\begin{tabular}{lllll}
\hline Macronutrients (g) & $\begin{array}{l}\text { Calories } \\
\text { (kcal) }\end{array}$ & CHO (g) & Fat (g) & PRO (g) \\
Pre-M1 (breakfast) & 528.0 & 81.2 & 11.6 & 24.7 \\
Pre-M2 & 499.6 & 103.6 & 3.2 & 14.1 \\
Pre-M3 & 469.5 & 97.1 & 4.3 & 10.6 \\
Pre-M4 & 398.3 & 60.9 & 9.1 & 18.2 \\
Post-M4 (dinner) & 1110.4 & 159.2 & 31.2 & 48.2 \\
Overall Macronutrient Intake (g) & N/A & 502.0 & 59.4 & 115.8 \\
Overall Energy Intake (kcal) & 3006 & 2008.0 & 534.6 & 463.2 \\
Percent of Energy Intake (\%) & Calories (\%) & CHO (\%) & Fat (\%) & PRO (\%) \\
Pre-M1 (breakfast) & 17.6 & 61.5 & 19.8 & 18.7 \\
Pre-M2 & 16.6 & 82.9 & 5.8 & 11.3 \\
Pre-M3 & 15.6 & 82.6 & 8.2 & 9.0 \\
Pre-M4 & 13.3 & 61.2 & 20.6 & 18.3 \\
Post-M4 (dinner) & 36.9 & 57.4 & 25.3 & 17.4 \\
Overall Energy Intake (\%) & 100.0 & 66.8 & 17.8 & 15.4 \\
\hline CHO: carbohydrate; PRO: protein; Pre-M1: before Taekwondo competition Match \\
\#1 (breakfast); Pre-M2: before Taekwondo competition Match \#2; Pre-M3: before \\
Taekwondo competition Match \#3; Pre-M4: before Taekwondo competition Match \\
\#4; Post-M4: after Taekwondo competition Match \#4 (completion of the \\
competition, dinner); N/A: Not applicable.
\end{tabular}

\section{Supplements}

All the vitamin supplements were purchased from GNC (General Nutrition Centers, Pittsburgh, PA, USA). Participants all received either Vit C+E (vitamin C: $2000 \mathrm{mg} / \mathrm{d}$; vitamin E [d-alpha tocopheryl]: $1400 \mathrm{U} / \mathrm{d}$; the daily dose of both Vit C and Vit $\mathrm{E}$ was split into two equal doses per day and provided at 0700 and 1700) or Placebo (comparable amounts of flour-based pill and soy-oil gel capsule), 
and both groups received same sized capsules and pills that looked identical to ensure double-blinding of treatments. At 3 days before the simulated matches, a blinded research team member provided the respective pills and capsules to each participants according to the study design. The supplementation consumption logs were also recorded by the research team member to ensure participants' compliance with supplement consumptions. On the TKD competition day, the morning supplements were provided at 0720 with breakfast, and the evening supplements were provided at 1730 with dinner after the final match (see Figure 1A for the supplementation timing during TKD match day).

\section{Analyses of biomarkers of muscle damage and hematological profiles}

A 10-mL venous blood sample was drawn from a forearm vein at 0700 in the morning (Pre-M1) and at 10-min before every match (Pre-M2, Pre-M3, and Pre-M4) and 24 hour after pre-M1 (Post-24h). Blood was collected into EDTA-treated tubes $(5 \mathrm{ml})$ or tubes with clot activator $(5 \mathrm{ml})$. Whole blood (EDTA) samples were used to determine hematological profiles, including red blood cell number (RBC), hematocrit (Hct), and blood platelet number (PLT using a hematology analyzer (RBC intra-assay $\mathrm{CV}=$ $1.5 \%$, Hct intra-assay CV $=1.5 \%$, PLT intra-assay CV $=$ $5 \%$; Sysmex XT-2000, Sysmex Corp., Kobe, Japan). The blood collected in tubes with clot activator were allowed to clot for $30 \mathrm{~min}$ then centrifuged at 3,000 $\times \mathrm{g}$ for $10 \mathrm{~min}\left(4^{\circ} \mathrm{C}\right)$ to obtain serum whereas EDTA tubes were centrifuged immediately after collection to obtain plasma. The intramuscular proteins, such as $\mathrm{CK}$ and myoglobin, can leak out from muscle cells when muscle damage occurs during or after intense exercise and are thus well-recognized as muscle damage biomarkers [20,21]. It is well established that muscle damage is associated with impaired muscle function and performance [22] and some studies have suggested that nutritional interventions can reduce muscle damage, which may be mechanistically linked to enhanced performance or recovery [23]. Therefore, monitoring circulating markers of muscle damage (CK and myoglobin) is of clinical relevance for determining the utility of strategies for promoting post-exercise recovery. We therefore selected these two biomarkers to reveal the severity of exercise-induced muscle damage during simulated TKD competition. Plasma creatine kinase (CK) was measured using an LX-20 clinical chemistry analyzer (intra-assay CV $=7.5 \%$; Beckman, Brea, CA, USA), and serum myoglobin was measured by radioimmunoassay using a test kit (intra-assay $\mathrm{CV}=$ 1.5\%; Daiichi Radioisotope Laboratory Ltd, Tokyo,
Japan) in accordance with the manufacturers' instructions.

\section{Statistical analysis}

The data were presented as mean \pm standard error of the mean (Mean \pm S.E.M.). IBM SPSS statistics for Windows version 19.0 statistical software (IBM Corp., Armonk, NY, USA) was used to perform statistical analyses. Prior to further statistical analysis, all data were examined for normality of distribution. The heart rate, blood lactate, blood creatine kinase levels, serum myoglobin concentrations, Hct, and hemolysis state were analyzed using 2-way (treatment group $x$ time) mixed analysis of variance (ANOVA, for repeated measures, between groups). The area under curve (AUC) of the variables listed above were compared between groups using independent $t$-test. For all measures, post hoc analysis was performed when significance was found using least significant difference. Pearson's correlational coefficient was used to determine the relationships between the AUC of platelet-to-lymphocyte ratio (PLR), the AUC of RBC loss (hemolysis), and the AUC of myoglobin. Differences were considered significant at $p<0.05$.

\section{Results}

\section{Heart rate and lactate responses during taekwondo matches}

Figure $1(B, C)$ displays the responses of heart rate and blood lactate concentrations during the 4 TKD simulated matches. The changes in heart rate and blood lactate concentrations significantly increased during TKD matches and rapidly declined during the rest between matches, and there were no differences in these two physiological parameters between Vit $\mathrm{C}+\mathrm{E}$ and PLA groups.

\section{Circulating myoglobin and creatine kinase levels}

Figure 2 displays the changes in myoglobin $(\mathrm{Mb})$ and creatine kinses (CK) in response to the TKD matches. For $\mathrm{Mb}$ there was a significant main effect of group $(p=0.002)$, time $(p<0.001)$ and group $x$ time interaction $(p=.040)$. Post hoc analyses revealed significantly lower $\mathrm{Mb}$ in the Vit $\mathrm{C}+\mathrm{E}$ group than PLA group at Pre M2, Pre M3 and Pre M4 (Pre M2 p = 0.040; Pre M3 $p=0.050$; Pre M4 $p=0.016)$. Mb AUC was also significantly lower in the Vit $\mathrm{C}+\mathrm{E}$ group compared to PLA group $(-47.0 \%, \mathrm{p}=0.021)$. For CK there was a significant main effect of time $(p<0.001)$ but there were no significant main effects of group $(p$ $=0.132)$ and interaction $(p=0.093)$. The Vit $C+E$ group showed significantly lower AUC of plasma CK than PLA group $(-57.5 \%, p=0.017)$. 


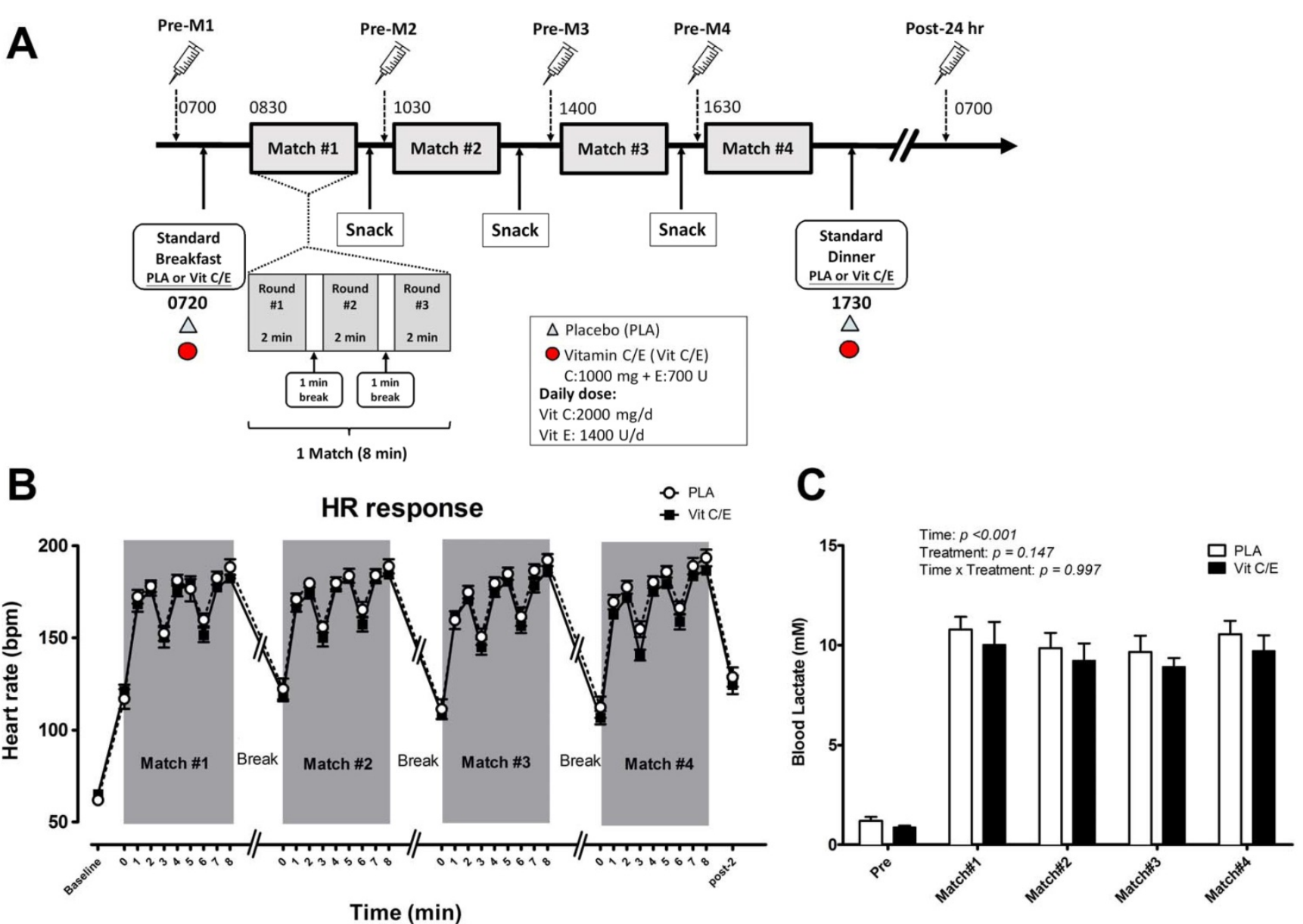

Figure 1. Experimental time-frame and physiological responses. (A) Experimental design of the match. The blood samples were drawn 10 min before each matchs (Pre-M1, Pre-M2, Pre-M3, and Pre-M4) and 24 hour after pre-M1 (Post-24h). (B) The heart rate (HR) of four serial matches (every match including 3 rounds, each round for 2 $\mathrm{min}$ and $1 \mathrm{~min}$ rest between every round) were recorded continuously. (C) The blood lactate levels were measured before match\#1 and after every match (PLA, open bar; Vit $\mathrm{C}+\mathrm{E}$, black bar).

A

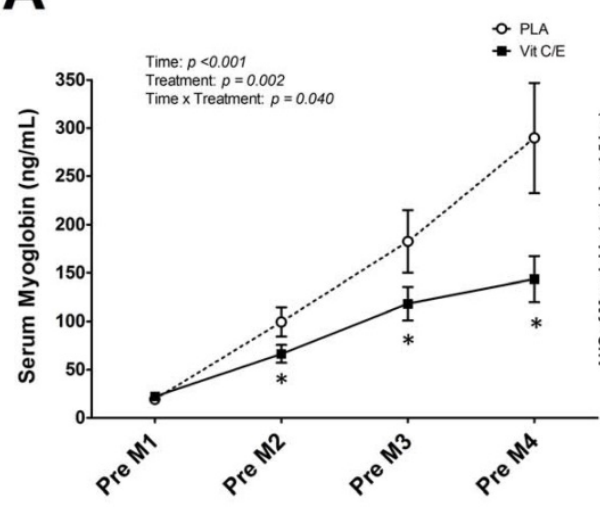

B

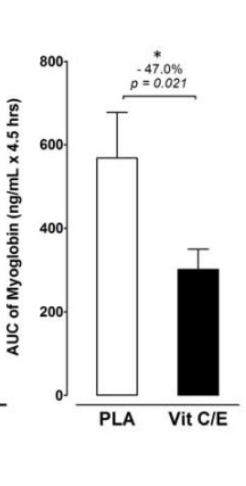

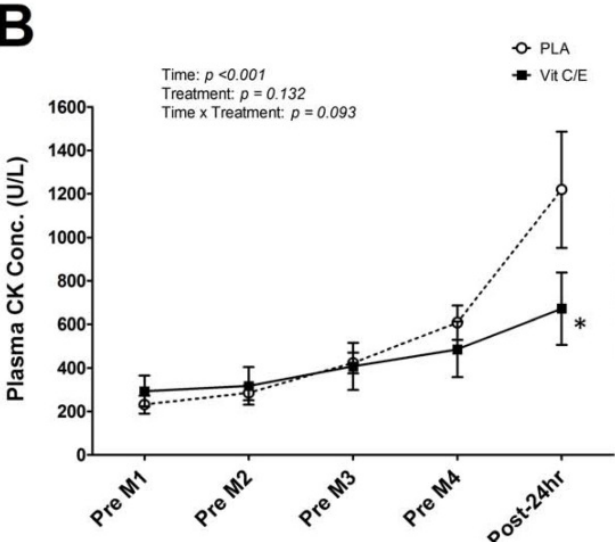

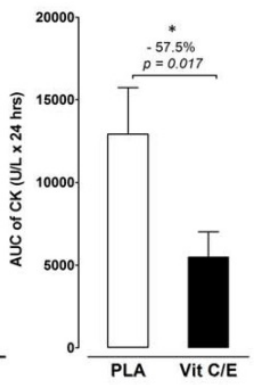

Figure 2. Muscle damage markers. (A) serum myoglobin $(\mathrm{Mb})$ and $(B)$ plasma creatine kinase $(C K)$ were measured before every matches (Pre-M1, Pre-M2, Pre-M3, and Pre-M4) and CK were measured at 24 hour after pre-Ml (Post-24h). The area under curve (AUC) of Mb and CK are also displayed in 2A and 2B, respectively (PLA, open bar; Vit $\mathrm{C}+\mathrm{E}$, black bar). Data are expressed as Mean \pm S.E.M. * significantly different from placebo $(p<0.05)$.

\section{Changes in hematocrit and hemolysis during taekwondo matches}

Hematological parameters are displayed in Figure 3. The hematocrit did not show any significant differences during simulated TKD matches, and there were no differences between the two groups (Fig. 3A).
For calculated hemolysis (i.e. the decrease in circulating RBCs) there was a significant main effect of group $(p=0.005)$, and time $(p<0.001)$ but no interaction $(\mathrm{p}=0.133)$ and the magnitude of hemolysis during TKD matches was significantly attenuated in the athletes with Vit $\mathrm{C}+\mathrm{E}$ supplementation (Fig. 3B). Similarly, the Vit C+E 
group also exhibited a significantly lower hemolysis AUC compared with those of PLA group $(p=0.034)$ (Fig. 3C).

\section{Circulating platelet-to-lymphocyte ratio (PLR)}

The platelet-to-lymphocyte ratio (PLR) was used to reflect changes in systemic inflammatory state during simulated matches [24]. For PLR there was a significant main effect of group $(\mathrm{p}=0.018)$ and time $(\mathrm{p}$ $<0.001)$ but no interaction $(\mathrm{p}=0.294)$. The Vit $\mathrm{C}+\mathrm{E}$ group exhibited a relatively lower response in the changes of PLR during simulated TKD matches (Fig. 4A; treatment effect: $p=0.018)$. Likewise, the AUC of PLR showed a trend to be lower in the Vit $\mathrm{C}+\mathrm{E}$ group compared to the PLA group $(\mathrm{p}=0.058)$ (Fig. 4B).

\section{Correlation analysis}

The AUC of PLR was positively correlated with the AUC of $\mathrm{Mb}$ (until the end of final TKD match) $(r=$
0.72, $\mathrm{p}<0.001$ ) (Fig. 5A). The AUC of PLR showed a trend for a negative correlation with the AUC of RBC loss during 24-hours from the beginning of the match day $(r=-0.40, p=.05)$ (Fig. 5B).

\section{Discussion}

To our knowledge, this study is the first to investigate the effects of antioxidant supplementation using the field-test model mimicking Olympic or international level Taekwondo competition. In the current double-blind, randomized controlled study, we found that a short-term (4-days) high-dose vitamin $C$ and $E$ supplementation (Vitamin C: 2000 $\mathrm{mg} / \mathrm{d}$; Vitamin E: $1400 \mathrm{U} / \mathrm{d}$ ) effectively attenuated the increases in circulating CK and myoglobin levels caused by successive TKD matches in the placebo group (Fig. 2). We also observed that vitamin C and E supplementation effectively suppressed the
A

Time: $p=017$

Treatment: $p=0.573$

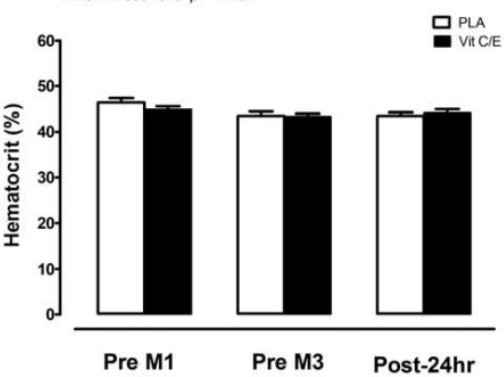

B

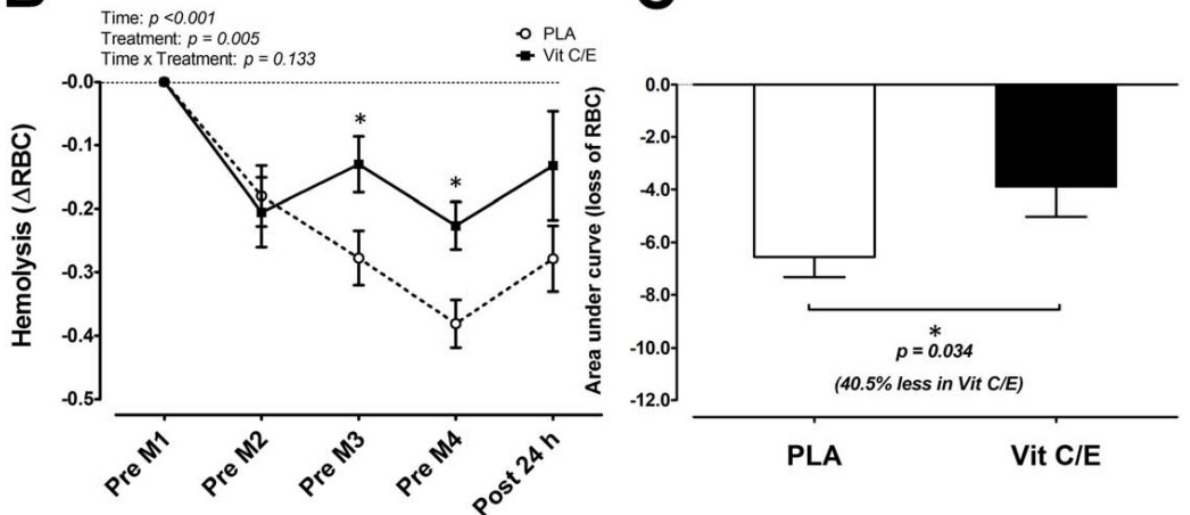

Figure 3. Hematocrit, hemolysis and red blood cell (RBC) counts. (A) Hematocrit at Pre-M1, Pre-M3, and Post-24h. (B) Hemolysis (calculated by change in RBC, $\triangle \mathrm{RBC}$ ) before each TKD match time point, and (C) the area under curve (AUC) in RBC loss (PLA, open bar; Vit C+E, black bar) in two groups. Data are expressed as Mean \pm S.E.M. * significantly different from placebo $(p<0.05)$.

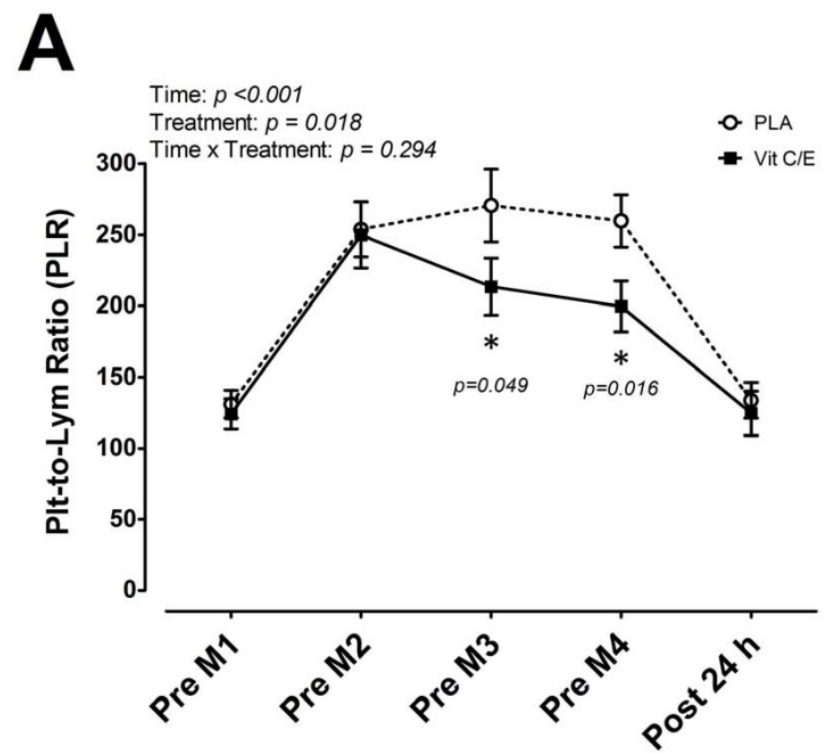

B

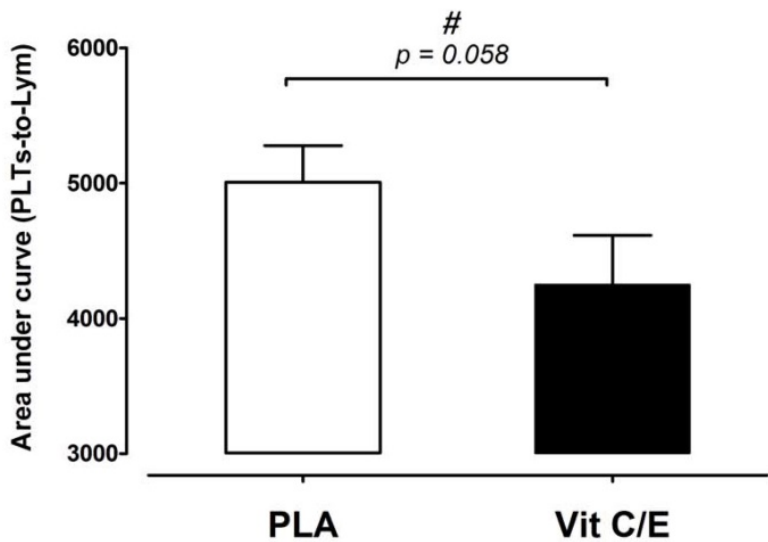

Figure 4. Systemic inflammatory response. (A) Platelet-to-lymphocyte Ratio (PLR). (B) PLR area under curve (AUC) i (PLA, open bar; Vit $C+E$, black bar). Data are expressed as Mean \pm S.E.M. * significantly different from placebo $(p<0.05)$. \# denotes approached significant trend $(p<0.075)$ between two groups $(p=0.058)$. 

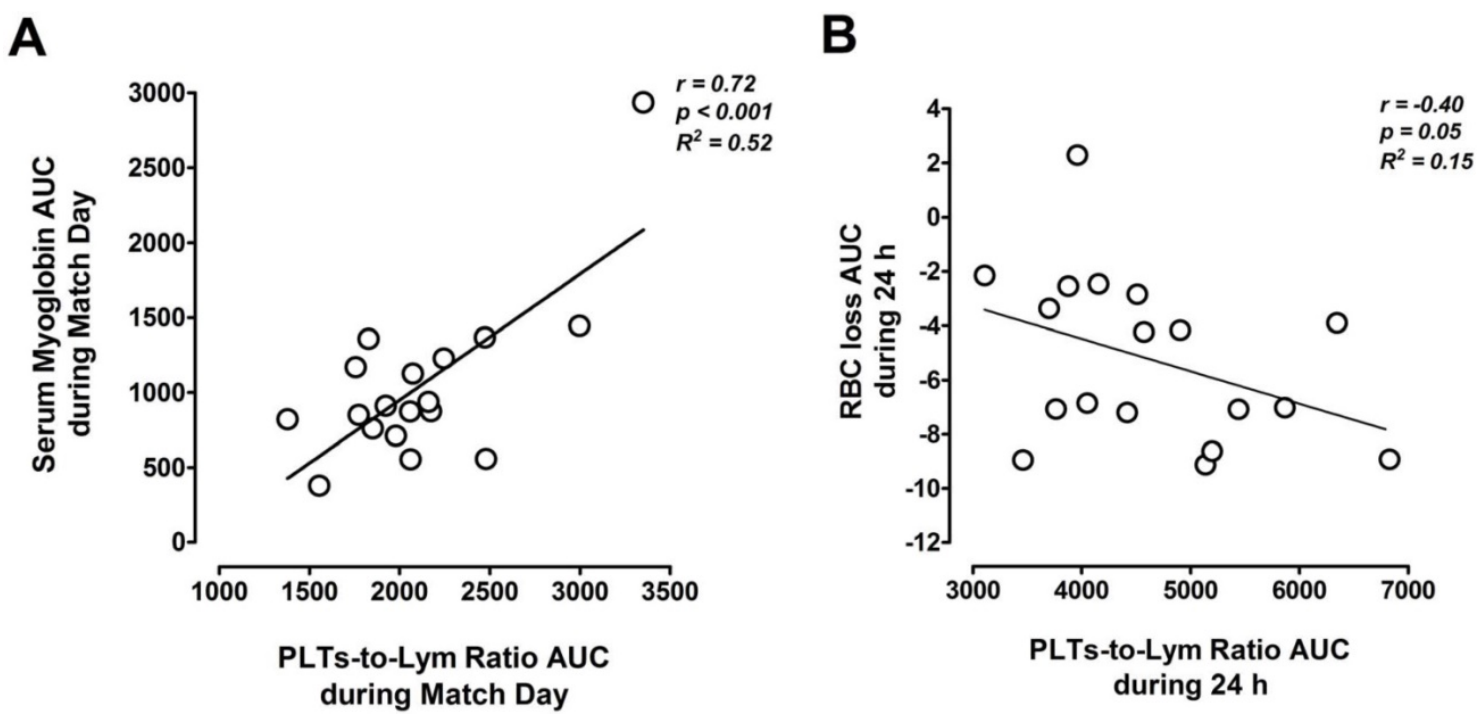

Figure 5. Correlation analysis. (A) correlation between AUC of PLR and AUC of Mb; (B) correlation between AUC of PLR and RBC loss AUC (hemolysis).

exercise-provoked red-blood-cell hemolysis (Fig. 3) and systemic inflammation (revealed by PLR) during TKD competition (Fig. 4). Furthermore, the degree of exercise-induced muscle damage appears to be associated with the magnitude of systemic inflammation after the TKD competition (Fig. 5). Short-term high-dose vitamin $\mathrm{C}$ and $\mathrm{E}$ supplementation demonstrated functional properties in terms of physiological protection and anti-inflammatory action. The combined benefits of physiological protection and anti-inflammatory action of vitamin $\mathrm{C}$ and $\mathrm{E}$ may potentially enhance recovery between matches.

Intensive exercise or competition can markedly increase acute muscle micro-damage and raise physiological stresses due to the high exercise intensity and large amount of eccentric movements $[18,19]$. During strenuous exercise consecutive eccentric mechanical force damages muscle cell membranes and alters membrane permeability, thereby resulting in the leaking of large amounts of intramuscular enzymes and proteins (e.g. CK, lactate dehydrogenase (LDH), and myoglobin, which reflect the degree of muscle damage) $[25,26]$. In addition the generation of reactive oxygen species may also contribute to muscle damage processes [27-29]. Previous studies also reported the functional bioactivities of vitamin $\mathrm{C}$ and vitamin $\mathrm{E}$ on attenuating exercise-induced muscle damage and oxidative cellular damage in response to various types of exercise, such as prolonged exercise, high-intensity exercise, and resistance training $[4,5$, 8]. Here we observed that the AUC values of both myoglobin $(p=.021)$ and $C K(p=.017)$ in the Vit $C+E$ group were significantly lower than that in PLA group, and this finding is consistent with previous studies investigating physiological responses during successive matches in other combative sport disciplines [30, 31]. In the current investigation, we also found that, compared with those in the placebo group, a short-term (4-days) high-dose vitamin $\mathrm{C}$ and E supplementation effectively attenuated the increases in muscle damage biomarkers caused by consecutive TKD competitions (Fig. 2A and 2B).

In this study, we observed that the intravascular number of red blood cells significantly decreased after the beginning of TKD competition, suggesting intravascular hemolysis during the consecutive TKD events. Strenuous exercise-induced red-blood cell hemolysis has been previously reported [32, 33], and hemolysis may result from intrinsic abnormalities of erythrocyte contents [34] or physical trauma in the circulation $[35,36]$. Also, exercise-induced oxidative stress appears to contribute to exercise-induced hemolysis [37], and the damaged erythrocyte membrane can lead to hemolysis due to the reduced cellular deforming capability and increased membrane rigidity [38, 39]. In the current study, short-term vitamin $\mathrm{C}$ and $\mathrm{E}$ supplementation significantly decreased the exercise-provoked red-blood-cell hemolysis (Fig. 3) compared to the placebo group even with the stable hematocrit levels. Moreover, the results of AUC in RBC loss showed that vitamin $C$ and $E$ supplementation exhibited better capacity to sustain the number of RBC throughout the TKD matches (Fig. 3C). Vitamin E, a lipid soluble antioxidant, shows the benefits on protecting plasma membrane integrity against multiple physical and chemical challenges [40,41], but there are several inconsistent findings [42, 43]. However, the discrepancy could be due to the usage of vitamin $\mathrm{E}$ alone but not combined with vitamin C $[42,43]$. 
Because vitamin $\mathrm{C}$ and vitamin $\mathrm{E}$ have been demonstrated to exert synergistic effects and have greater biological functions in concert [5-7], thus we speculate that the benefits on attenuated exercise-induced RBC hemolysis could be due to the combination of these two antioxidants.

Antioxidant supplements are generally used by athletes as ergogenic aids to counteract the oxidative stress of exercise. During intensive TKD competitions, the athletes have to experience numerous eccentric movements from activities such as jumping, rapid moving, kicking and landing, and eccentric exercise components can damage muscle tissue and provoke acute inflammatory responses [44, 45]. In this study, the correlations between platelet-to-lymphocyte ratio (PLR; systemic inflammation marker) and serum myoglobin level (Fig. 5A) and RBC hemolysis (Fig. 5B) suggest that the increased inflammatory response and tissue damage reflects the accumulative stress on the membrane structural changes of erythrocyte and skeletal muscle during exercise. More importantly, we demonstrate that short-term vitamin $\mathrm{C}$ and $\mathrm{E}$ supplementation significantly ameliorated the increased levels of muscle damage, inflammatory response, and RBC hemolysis resulting from successive TKD matches, which is in line with previous findings about the anti-inflammatory and anti-oxidant effects in response to heavy exertion [46-48]. Taken together, we speculate the mechanisms by which short-term Vit $\mathrm{C}+\mathrm{E}$ may prevent or reduce muscle damage is through the quenching effects of these antioxidants suppressing exercise-induced oxidative stress $[3,49,50]$.

It is important to note, however, some recent evidence suggests that the exercise-induced increase in reactive oxygen species (ROS) is necessary for muscle adaptation to occur during exercise training [51] and that chronic anti-oxidant treatment may hinder chronic training adaptions [10-12, 52]. However, in competitive situations where the aim is to optimize performance in the present event, stimulating chronic adaptation is not the main priority. Hence a short-term benefit in muscle damage and inflammatory profile may translate to acute benefits to recovery and performance. Furthermore, the relatively short supplementation period (only 3 days before and on the day of competition) used in the present study is less likely to perturb training adaptions compared to the longer-term high dose supplementation periods used in some previous studies, although this will required further investigation.

Official TKD matches consist of short bouts of near maximal-intensity movements $(2 \mathrm{~min} \times 3$ rounds) with short 1-min intervals between rounds
[15], thus the capacity of rapid recovery from exhaustive exercise is critical in this combative sport. The aerobic system is important to sustain high-intensity activity and facilitate recovery between consecutive bouts during competitive events [53-55], as higher aerobic capacity is highly associated with greater recovery capacity after maximal exercise [56]. This emphasizes the importance of keeping RBC intact. Because the loss of RBC may alter oxygen transport capacity [57], the exercise-induced hemolysis might impair aerobic capacity and recovery. Together with our current findings and previous evidence, we suggest that short-term vitamin $C$ and $E$ supplementation is effective for protecting the integrity of RBC and reducing muscle damage in response to intensive Taekwondo competition.

\section{Limitations}

There is a considerable difference in body mass between the smallest and largest athletes in the present study meaning the relative (to body mass) doses may differ. However, each athlete was pair matched with a similar sized athlete meaning there was an equal distribution between groups. Moreover, similar to our experimental design, several studies supplemented vitamin $C$ or vitamin $E$ in absolute dosages to investigate the protective effects on muscle damage, oxidative stress, and muscle function following an acute bout of high-intensity exercise [4, $5,8,9]$, which is in line with reference intake values (and upper intake limits), which are typically given as an absolute dose. Therefore, we used commercially available products made in fixed absolute doses, which is also practically relevant to the real world scenarios of both coaches and athletes. We did not measure participants' initial aerobic capacity and pre-supplementation dietary intake and blood parameters so we cannot completely rule out the possible impacts of initial physiological levels on our outcome measurements. However, all athletes were elite and competitive in the same division so we do not expect significant differences between participants. We also attempted to minimize possible confounding factors through instructing participants to maintain their habitual diet according to general dietary guidelines and light physical activity level during the study.

\section{Conclusion}

Short-term (4-days) supplementation with high dose vitamin C and E (Vitamin C: $2000 \mathrm{mg} / \mathrm{d}$; Vitamin E: $1400 \mathrm{U} / \mathrm{d}$ ) effectively attenuated exercise-induced tissue damage and inflammatory response during and after 4 consecutive TKD matches 
on the same day. This may contribute to the enhancement of performance in events that require repeated maximal exertion with short recovery periods to be repeated numerous times in a single day, such as competitive combat sports. Future studies are warranted to investigate the effects of short-term vitamin $\mathrm{C}$ and $\mathrm{E}$ supplementation on muscle damage, inflammation and also performance and recovery between matches using this field-test model.

\section{Abbreviations}

TKD: Taekwondo; Vit: vitamin; WTF: World Taekwondo Federation; HR: heart rate; RBC: red blood cell; Hct: hematocrit; PLT: blood platelet number; CK: creatine kinase; AUC: area under curve; $\mathrm{Mb}$ : myoglobin; PLR: Platelet-to-lymphocyte ratio; LDH: lactate dehydrogenase; ROS: reactive oxygen species.

\section{Acknowledgements}

All authors would like to show a deep appreciations to all athletes for their participating in this study. This work was supported by the Ministry of Science and Technology, Taiwan, ROC Grant number 105-2410-H-227-004. We also sincerely thank the National Taipei University of Technology and National Taipei University of Nursing and Health Sciences for providing us with necessary resources and administration support throughout the study.

\section{Author Contributions}

Conceived and designed the experiments: CCC, and YHL. Performed the experiments: CCC, YCS, CYC, and YHL. Analyzed and discussed the data: CCC, GD, and YHL. Contributed reagents/materials/analysis tools: CCC, YCS, CYC, and YHL. Wrote the paper: CCC, GD, and YHL. All authors read and approved the final form of this work.

\section{Ethics approval and consent to participate}

The protocol was approved by Institute Review Board (IRB) of the University of Taipei and all ethical guidelines for human experimentation were followed. Participants completed a written informed consent before the experiment.

\section{Competing Interests}

The authors have declared that no competing interest exists.

\section{References}

1. Beyer RE. The role of ascorbate in antioxidant protection of biomembranes: interaction with vitamin E and coenzyme Q. J Bioenerg Biomembr. 1994; 26: 349-58.
2. Burton GW, Traber MG. Vitamin E: antioxidant activity, biokinetics, and bioavailability. Annu Rev Nutr. 1990; 10: 357-82.

3. Itoh $\mathrm{H}$, Ohkuwa T, Yamazaki $\mathrm{Y}$, Shimoda T, Wakayama A, Tamura S, et al. Vitamin $\mathrm{E}$ supplementation attenuates leakage of enzymes following 6 successive days of running training. Int J Sports Med. 2000; 21: 369-74.

4. Bryer SC, Goldfarb AH. Effect of high dose vitamin C supplementation on muscle soreness, damage, function, and oxidative stress to eccentric exercise. J Sport Nutr Exerc Metab. 2006; 16: 270-80.

5. Rokitzki L, Logemann E, Sagredos AN, Murphy M, Wetzel-Roth W, Keul J. Lipid peroxidation and antioxidative vitamins under extreme endurance stress*. Acta Physiol Scand. 1994; 151: 149-58.

6. Rinne T, Mutschler E, Wimmer-Greinecker G, Moritz A, Olbrich HG. Vitamins $\mathrm{C}$ and $\mathrm{E}$ protect isolated cardiomyocytes against oxidative damage. Int $\mathrm{J}$ Cardiol. 2000; 75: 275-81.

7. Niki E, Noguchi N, Tsuchihashi H, Gotoh N. Interaction among vitamin C, vitamin E, and beta-carotene. Am J Clin Nutr. 1995; 62: 1322S-6S.

8. Taghiyar M, Darvishi L, Askari G, Feizi A, Hariri M, Mashhadi NS, et al. The Effect of Vitamin C and E Supplementation on Muscle Damage and Oxidative Stress in Female Athletes: A Clinical Trial. Int J Prev Med. 2013; 4: S16-S23.

9. Shafat A, Butler P, Jensen RL, Donnelly AE. Effects of dietary supplementation with vitamins $\mathrm{C}$ and $\mathrm{E}$ on muscle function during and after eccentric contractions in humans. Eur J Appl Physiol. 2004; 93: 196-202.

10. Gomez-Cabrera M-C, Domenech E, Romagnoli M, Arduini A, Borras C, Pallardo FV, et al. Oral administration of vitamin $\mathrm{C}$ decreases muscle mitochondrial biogenesis and hampers training-induced adaptations in endurance performance. Am J Clin Nutr. 2008; 87: 142-9.

11. Paulsen G, Cumming KT, Holden G, Hallén J, Rønnestad BR, Sveen O, et al. Vitamin $C$ and $E$ supplementation hampers cellular adaptation to endurance training in humans: a double-blind, randomised, controlled trial. J Physiol. 2014; 592: 1887-901.

12. Morrison D, Hughes J, Della Gatta PA, Mason S, Lamon S, Russell AP, et al. Vitamin $\mathrm{C}$ and $\mathrm{E}$ supplementation prevents some of the cellular adaptations to endurance-training in humans. Free Radic Biol Med. 2015; 89: 852-62.

13. Yfanti C, Akerstrom T, Nielsen S, Nielsen AR, Mounier R, Mortensen $\mathrm{OH}$, et al. Antioxidant supplementation does not alter endurance training adaptation. Med Sci Sports Exerc. 2010; 42: 1388-95.

14. Melhim A. Aerobic and anaerobic power responses to the practice of taekwon-do. Br J Sports Med. 2001; 35: 231-4

15. Bridge CA, Ferreira da Silva Santos J, Chaabene H, Pieter W, Franchini E. Physical and physiological profiles of taekwondo athletes. Sports Med. 2014; 44: 713-33

16. Kazemi M, Casella C, Perri G. 2004 Olympic Tae Kwon Do Athlete Profile. J Can Chiropr Assoc. 2009; 53: 144-52.

17. Markovic G, Misigoj-Durakovic M, Trninic S. Fitness profile of elite Croatian female taekwondo athletes. Coll Antropol. 2005; 29: 93-9.

18. Kon M, Tanabe K, Lee H, Kimura F, Akimoto T, Kono I. Eccentric muscle contractions induce greater oxidative stress than concentric contractions in skeletal muscle. Appl Physiol Nutr Metab. 2007; 32: 273-81.

19. Chiodo S, Tessitore A, Cortis C, Cibelli G, Lupo C, Ammendolia A, et al. Stress-related hormonal and psychological changes to official youth Taekwondo competitions. Scand J Med Sci Sports. 2011; 21: 111-9.

20. Twist $C$, Eston $R$. The effects of exercise-induced muscle damage on maximal intensity intermittent exercise performance. Eur J Appl Physiol. 2005; 94: $652-8$.

21. Clarkson PM, Kearns AK, Rouzier P, Rubin R, Thompson PD. Serum creatine kinase levels and renal function measures in exertional muscle damage. Med Sci Sports Exerc. 2006; 38: 623-7.

22. Byrne C, Twist C, Eston R. Neuromuscular function after exercise-induced muscle damage: theoretical and applied implications. Sports Med. 2004; 34: $49-69$

23. Saunders MJ, Kane MD, Todd MK. Effects of a carbohydrate-protein beverage on cycling endurance and muscle damage. Med Sci Sports Exerc. 2004; 36: 1233-8.

24. Turkmen K, Erdur FM, Ozcicek F, Ozcicek A, Akbas EM, Ozbicer A, et al. Platelet-to-lymphocyte ratio better predicts inflammation than neutrophil-to-lymphocyte ratio in end-stage renal disease patients. Hemodial Int. 2013; 17: 391-6.

25. Brancaccio P, Maffulli N, Limongelli FM. Creatine kinase monitoring in sport medicine. Br Med Bull. 2007; 81: 209-30.

26. Warren GL, Ingalls CP, Lowe DA, Armstrong RB. Excitation-contraction uncoupling: major role in contraction-induced muscle injury. Exerc Sport Sci Rev. 2001; 29: 82-7.

27. Aoi W, Naito $Y$, Takanami $Y$, Kawai $Y$, Sakuma K, Ichikawa H, et al. Oxidative stress and delayed-onset muscle damage after exercise. Free Radic Biol Med. 2004; 37: 480-7.

28. Powers SK, Nelson WB, Hudson MB. Exercise-induced oxidative stress in humans: cause and consequences. Free Radic Biol Med. 2011; 51: 942-50.

29. Chevion S, Moran DS, Heled Y, Shani Y, Regev G, Abbou B, et al. Plasma antioxidant status and cell injury after severe physical exercise. Proc Natl Acad Sci. 2003; 100: 5119-23.

30. Brandão F, Fernandes HM, Alves JV, Fonseca S, Reis VM. Hematological and biochemical markers after a Brazilian Jiu-Jitsu tournament in world-class athletes. Rev Bras Cineantropom Desempenho Hum. 2014; 16: 144-51. 
31. Detanico D, Dal Pupo J, Franchini E, Dos Santos SG. Effects of successive judo matches on fatigue and muscle damage markers. J Strength Cond Res. 2015; 29: 1010-6.

32. Gilligan DR, Altschule MD, Katersky EM. Physiological intravascular hemolysis of exercise. Hemoglobinemia and hemoglobinuria following cross-country runs. J Clin Invest. 1943; 22: 859-69.

33. Robinson $Y$, Cristancho E, Böning D. Intravascular hemolysis and mean red blood cell age in athletes. Med Sci Sports Exerc. 2006; 38: 480-3.

34. Beutler E, Luzzatto L. Hemolytic anemia. Semin Hematol. 1999; 36: 38-47.

35. Berkowitz FE. Hemolysis and infection: categories and mechanisms of their interrelationship. Rev Infect Dis. 1991; 13: 1151-62.

36. Engelfriet CP. The immune destruction of red cells. Transfus Med. 1992; 2: 1-6.

37. Senturk UK, Gunduz F, Kuru O, Kocer G, Ozkaya YG, Yesilkaya A, et al. Exercise-induced oxidative stress leads hemolysis in sedentary but not trained humans. J Appl Physiol. 2005; 99: 1434-41.

38. Kiefer CR, Snyder LM. Oxidation and erythrocyte senescence. Curr Opin Hematol. 2000; 7: 113-6.

39. Liu SC, Zhai S, Lawler J, Palek J. Hemin-mediated dissociation of erythrocyte membrane skeletal proteins. J Biol Chem. 1985; 260: 12234-9.

40. Quintanilha AT, Packer L, Davies JMS, Racanelli TL, Davies KJ. Membrane effects of vitamin $\mathrm{E}$ deficiency: bioenergetic and surface charge density studies of skeletal muscle and liver mitochondria. Ann N Y Acad Sci. 1982; 393: 32-47.

41. Davies KJA, Quintanilha AT, Brooks GA, Packer L. Free radicals and tissue damage produced by exercise. Biochem Biophys Res Commun. 1982; 107: 1198-205.

42. Avery NG, Kaiser JL, Sharman MJ, Scheett TE, Barnes DM, Gomez AL, et al. Effects of vitamin E supplementation on recovery from repeated bouts of resistance exercise. J Strength Cond Res. 2003; 17: 801-9.

43. Siciliano P, Parker A, Lawrence L. Effect of dietary vitamin E supplementation on the integrity of skeletal muscle in exercised horses. J Anim Sci. 1997; 75: 1553-60.

44. Faulkner JA, Brooks SV, Opiteck JA. Injury to Skeletal Muscle Fibers During Contractions: Conditions of Occurrence and Prevention. Phys Ther. 1993; 73: 911-21.

45. Toft AD, Jensen LB, Bruunsgaard H, Ibfelt $T$, Halkjær-Kristensen J, Febbraio $\mathrm{M}$, et al. Cytokine response to eccentric exercise in young and elderly humans. Am J Physiol Cell Physiol. 2002; 283: C289-95.

46. Nieman DC, Peters EM, Henson DA, Nevines EI, Thompson MM. Influence of vitamin C supplementation on cytokine changes following an ultramarathon. J Interferon Cytokine Res. 2000; 20: 1029-35.

47. Peters E, Anderson R, Nieman D, Fickl H, Jogessar V. Vitamin C supplementation attenuates the increases in circulating cortisol, adrenaline and anti-inflammatory polypeptides following ultramarathon running. Int $\mathrm{J}$ Sports Med. 2001; 22: 537-43.

48. Peters $\mathrm{E}$, Anderson R, Theron A. Attenuation of increase in circulating cortisol and enhancement of the acute phase protein response in vitamin C-supplemented ultramarathoners. Int J Sports Med. 2001; 22: 120-6.

49. Bryer SC, Goldfarb AH. Effect of high dose vitamin C supplementation on muscle soreness, damage, function, and oxidative stress to eccentric exercise. Int J Sport Nutr Exerc Metab. 2006; 16: 270-80.

50. Taghiyar M, Darvishi L, Askari G, Feizi A, Hariri M, Mashhadi NS, et al. The effect of vitamin $C$ and e supplementation on muscle damage and oxidative stress in female athletes: a clinical trial. Int J Prev Med. 2013; 4: S16-23.

51. Urso ML, Clarkson PM. Oxidative stress, exercise, and antioxidant supplementation. Toxicology. 2003; 189: 41-54.

52. Nikolaidis MG, Kerksick CM, Lamprecht M, McAnulty SR. Does vitamin C and $\mathrm{E}$ supplementation impair the favorable adaptations of regular exercise? Oxid Med Cell Longev. 2012; 2012: 707941.

53. Bridge CA, Jones MA, Drust B. Physiological responses and perceived exertion during international Taekwondo competition. Int J Sports Physiol Perform. 2009; 4: 485-93.

54. Campos FA, Bertuzzi R, Dourado AC, Santos VG, Franchini E. Energy demands in taekwondo athletes during combat simulation. Eur J Appl Physiol. 2012; 112: 1221-8.

55. Chiodo S, Tessitore A, Cortis C, Lupo C, Ammendolia A, Iona T, et al. Effects of official Taekwondo competitions on all-out performances of elite athletes. J Strength Cond Res. 2011; 25: 334-9.

56. Ostojic SM, Stojanovic MD, Calleja-Gonzalez J. Ultra short-term heart rate recovery after maximal exercise: relations to aerobic power in sportsmen. Chin J Physiol. 2011; 54: 105-10.

57. Hillman SS, Withers PC, Hedrick MS, Kimmel PB. The effects of erythrocythemia on blood viscosity, maximal systemic oxygen transport capacity and maximal rates of oxygen consumption in an amphibian. J Comp Physiol B, Biochem Syst Environ Physiol. 1985; 155: 577-81. 